\title{
Induced sputum in the management of COPD: clinical implications
}

\author{
A. Zanini ${ }^{1}$, S. Della Patrona1 , A.L. Facchini ${ }^{1}$, A. Spanevello1,2
}

ABSTRACT: Induced sputum in the management of COPD: clinical implications. A. Zanini, S. Della Patrona, A.L. Facchini, A. Spanevello.

Sputum induction can be used as a non-invasive technique to investigate airway inflammation in asthma and COPD. We reported the case of a 68 year old man with COPD, stage III GOLD, that underwent sputum induction during two exacerbation episodes. The first cell count showed a typical sputum neutrophilia, whereas the second showed sputum eosinophilia. On the basis of sputum cellularity, we decided to treat the first episode with a course of antibiotics and the second exacerbation with a course of antibiotics and oral steroids. The patient showed improvement in both cases, obtaining clinical stabilisation. The induced sputum cell count could be a useful technique in a clinical setting to evaluate the cellular characteristics of airway inflammation during COPD exacerbation and modulate the antinflammatory therapy. Monaldi Arch Chest Dis 2012; 77: 1, 23-25.

Keywords: COPD, Airway inflammation, Induced sputum.

1 Division of Pneumology, Fondazione S. Maugeri, IRCCS Rehabilitation Institute of Tradate (Varese),

2 Department of Respiratory Diseases, University of Insubria, Varese, Italy.

Correspondence: Dr. Andrea Zanini, Fondazione Salvatore Maugeri, Via Roncaccio 16, 21049 Tradate (VA), Italy; e-mail: andrea.zanini@fsm.it

\section{Introduction}

COPD has been increasingly recognised as a chronic inflammatory disease characterised by sputum neutrophilia and, in some cases, eosinophilia [1]. Use of induced sputum cell counts has been largely proposed as a non-invasive technique to investigate airway inflammation in asthma and COPD [2, 3]. The technique of sputum induction allows the study of COPD patients during exacerbation as it is safe and well tolerated [4]. Neutrophilic inflammation is further increased during exacerbation [5]. Moreover, the presence of eosinophilic inflammation can be detected during exacerbation in subjects with COPD. Sputum eosinophilia identifies a subgroup of patients who particularly respond to corticosteroid treatment [6].

\section{Case Report}

A 68 year old man attended for routine review of his COPD complaining that he had recently been more breathless. His record reported a concomitant history of hypertension and hypercholesterolemia. He had a full-time commercial job with his wife. The man smoked ten cigarettes per day; he had a 25 pack-year history of smoking. He tried to quit smoking with the aid of nicotine patch, but his periods of abstinence had been short. He had two courses of antibiotics and oral steroids for exacerbations in the last 12 months. He produced copious sputum and chronic cough in the mornings. Current medications includes a salmeterol/fluticasone com- bination inhaler, a tiotropium inhaler, ramipril, atorvastatin. On physical examination the lung sounded hyperinflated and had decreased breath sounds with some bilateral wheezes. The heart sounds were normal. A chest x-ray showed hyperinflation. Spirometry result: FVC $3.36 \mathrm{~L}$ ( $89 \%$ of predicted value); $\mathrm{FEV}_{1} 1.38 \mathrm{~L}$ (47\% pred.). Oxygen saturation was $96 \%$. Induced sputum cell count at baseline: neutrophils 78\%, eosinophils 3\%.

Three months later the man attended to the out-patient office for urgent visit for COPD exacerbation (increase cough, increase purulent yellow/green sputum production, increased breathless). There were some crackles without wheezes on thoracic examination. Spirometry result: FVC $3.18 \mathrm{~L}$ (84\% pred.); $\mathrm{FEV}_{1} 1.20 \mathrm{~L}$ (41\% pred.). Oxygen saturation was $92 \%$. Induced sputum cell count: neutrophils $95 \%$, eosinophils $1 \%$ (fig. 1). The man was treated with a course of antibiotics (Levofloxacin $500 \mathrm{mg} / \mathrm{die}$ - seven days) without oral steroids and continued his therapy with inhaled bronchodilators and steroids. Symptoms and the sputum purulence were reduced after 10 days; oxygen saturation was $95 \%$.

Seven months later the man attended the ER for serious breathless and wheezing. The entity and the colour of the sputum did not significantly change. On physical examination there were an increased sounds mainly wheezes. Spirometry was not done and oxygen saturation was $90 \%$. The patient was treated with inhaled salbutamol + ipratropium and theophylline + corticosteroids i.v. and a prescription of antibiotics (Levofloxacin 500 $\mathrm{mg} /$ day). The day after he attended our lab and 


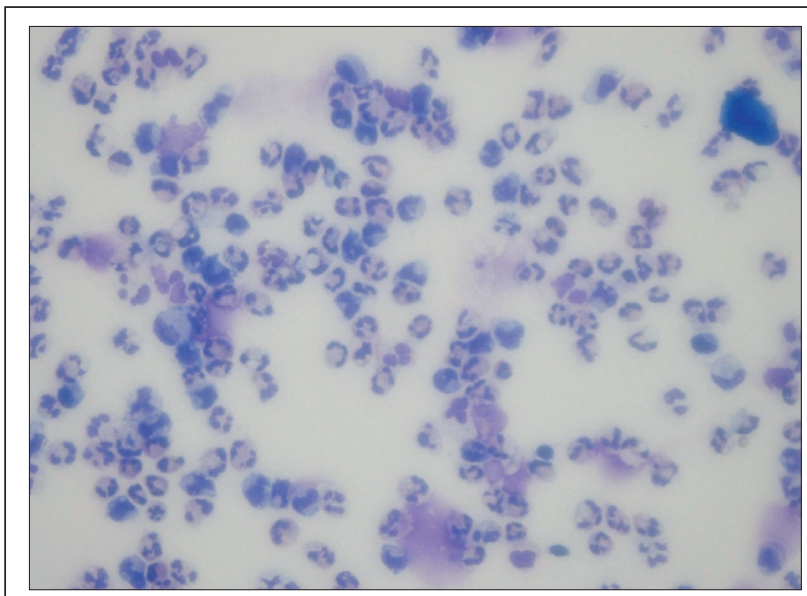

Fig. 1. - Induced sputum showing a high percentage of neutrophils (H \& E Stain, 10X).

spirometry + induced sputum were performed. Spirometry result: FVC 3.40 L (90\% pred.); $\mathrm{FEV}_{1} 1.29 \mathrm{~L}$ (44\% pred.). Oxygen saturation was $94 \%$. Induced sputum cell count: neutrophils $74 \%$, eosinophils $11 \%$ (fig. 2). The main causes of systemic eosinophilia - such as allergies (including allergic rhinitis and nasal polyposis), parasitic infections, neoplasms and immune disorders - were excluded. A course of oral steroids was started (Prednisone $25 \mathrm{mg} /$ die for seven days, then $12.5 \mathrm{mg} / \mathrm{die}$ for five days). Cough and wheezing were largely reduced after five days of oral steroids treatment.

One month later a sputum induction was performed: neutrophils $81 \%$, eosinophils $3 \%$. A schematic diagram of the case history reporting the clinical phases and the percentages of sputum cells is reported in figure 3 .

The induced sputum cell count could be a useful technique in a clinical setting to evaluate the cellular characteristics of airway inflammation during COPD exacerbation and modulate the antiinflammatory therapy.

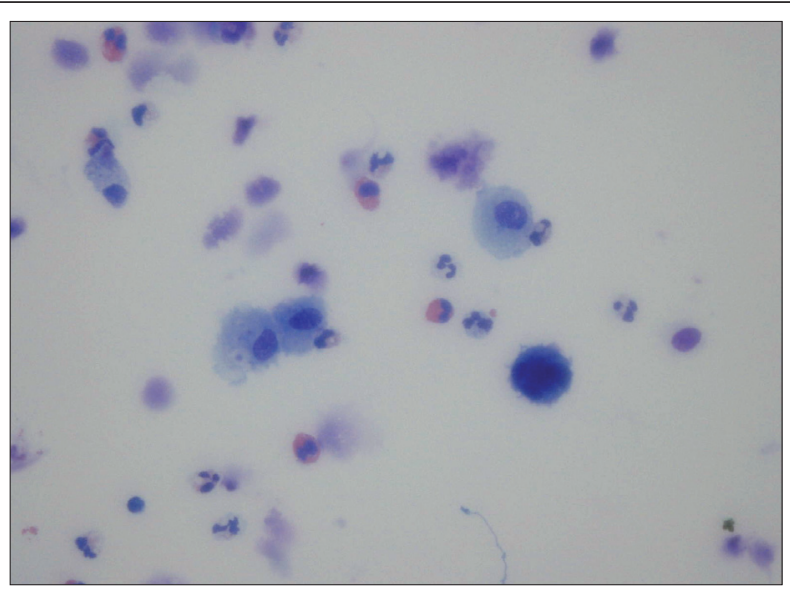

Fig. 2. - Induced sputum showing a high percentage of eosinophils (H \& E Stain, 10X).

\section{Discussion}

While chronic asthma is characterised by increased numbers of eosinophils in the sputum, COPD is characterised by increased numbers of neutrophils, although an increase in the numbers of neutrophils may also found in asthmatic patients $[2,3]$. Many inflammatory mediators have been analysed in the fluid-phase of induced sputum. Several cytokines, chemokines, eicosanoids, markers of oxidative stress show an increase in COPD compared to asymptomatic smokers, with a further increase during exacerbations. Moreover, sputum fluid-phase in COPD shows an increase in neutrophilic granulocyte proteins and others molecules, that represent markers of neutrophil activation [5]. All these findings indicate that neutrophils are relevant in the pathogenesis of COPD, and there is a negative correlation between neutrophil count and the decline of $\mathrm{FEV}_{1}$ [7].

We can use induced sputum to monitor inflammation in COPD. Interestingly, sputum cells were analysed on three separate occasions at fourweekly intervals [3]. Moderate, clinically stable COPD was associated with stable induced-sputum inflammatory markers, and the quantification of most cellular and soluble components is sufficiently reproducible [3]. Serial monitoring of inflammation in COPD using surrogate markers of induced sputum is a suitable tool for clinical routine, research purposes, and clinical trials.

Additionally, sputum induction can be safely carried out in patients with mild-tomoderate COPD who 
experienced an exacerbation, and this occurs with no greater risk than in stable patients with COPD. This finding is very important, considering that acute exacerbations play an important role on the natural history of COPD. Bathoorn et al. performed sputum induction in COPD patients, both in the stable phase and during exacerbation [4]. In this study, the median $\mathrm{FEV}_{1}$ for the stable phase and exacerbation were $61 \%$ and $51 \%$ of predicted, respectively, and the median decrease in $\mathrm{FEV}_{1}$ with sputum induction during an exacerbation was 0.271 vs 0.281 during the stable phase [4].

Although COPD is mainly a neutrophilic airway disease, a substantial part of patients fitting the functional definition of COPD exhibit significant sputum eosinophilia $(>3 \%)$ [8]. There is increasing evidence that the presence of sputum eosinophilia predicts an objective response to oral and inhaled corticosteroid treatment in COPD [6, 8-10]. Brightling et al. reported that the response in term of lung function to a two-week course of oral prednisolone increased as the baseline sputum eosinophilia count increased, and was associated with a marked treatment-induced fall in the sputum eosinophil count, but no change in sputum markers of neutrophilic inflammation [6]. Moreover, Siva et al. showed that a management approach over a 12-month period with the additional aim of reducing the sputum eosinophil count $<3 \%$ using corticosteroids was associated with a $62 \%$ reduction in severe exacerbation of COPD requiring hospitalisation when compared to traditional symptom-based management [9]. Based on these reports, a measurement of sputum eosinophil counts could be used to identify COPD patients with corticosteroids responsive disease and to guide treatment.

\section{References}

1. Lacoste JY, Bousquet J, Chanez P, et al. Eosinophilic and neutrophilic inflammation in asthma, chronic bronchitis, and chronic obstructive pulmonary disease. $J$ Allergy Clin Immunol 1993; 92: 537-548.

2. Spanevello A, Migliori GB, Sharara A, et al. Induced sputum to assess airway inflammation: a study of reproducibility. Clin Exp Allergy 1997; 27: 1138-1144.

3. Beeh KM, Beier J, Kornmann O, Mander A, Buhl R. Long-term repeatability of induced sputum cells and inflammatory markers in stable, moderately severe COPD. Chest 2003; 123: 778-783.

4. Bathoorn E, Liesker J, Postma D, Koëter G, van Oosterhout AJ, Kerstjens HA. Safety of sputum induction during exacerbations of COPD. Chest 2007; 131: 432438.

5. Bhowmik A, Seemungal TAR, Sapsford RJ, Wedzicha JA. Relation of sputum inflammatory markers to symptoms and lung function changes in COPD exacerbations. Thorax 2000; 55: 114-120.

6. Brightling CE, Monteiro W, Ward R, et al. Sputum eosinophilia and short-term response to prednisolone in chronic obstructive pulmonary disease: a randomised controlled trial. Lancet 2000; 356: 1480-1485.

7. Stanescu D, Sanna A, Veriter C, et al. Airawys obstruction, chronic expectoration, and rapid decline of $\mathrm{FEV}_{1}$ in smokers are associated with increased levels of sputum neutrophils. Thorax 1996; 51: 267-271.

8. Pizzichini E, Pizzichini MM, Gibson P, et al. Sputum eosinophilia predicts benefit from prednisone in smokers with chronic obstructive bronchitis. Am J Respir Crit Care Med 1998; 158: 1511-1517.

9. Siva R, Green RH, Brightling CE, et al. Eosinophilic airway inflammation and exacerbations of COPD: a randomised controlled trial. Eur Respir J 2007; 29: 906-913.

10. Brightling CE, McKenna S, Hargadon B, et al. Sputum eosinophilia and the short term response to inhaled mometasone in chronic obstructive pulmonary disease. Thorax 2005; 60: 193-198.

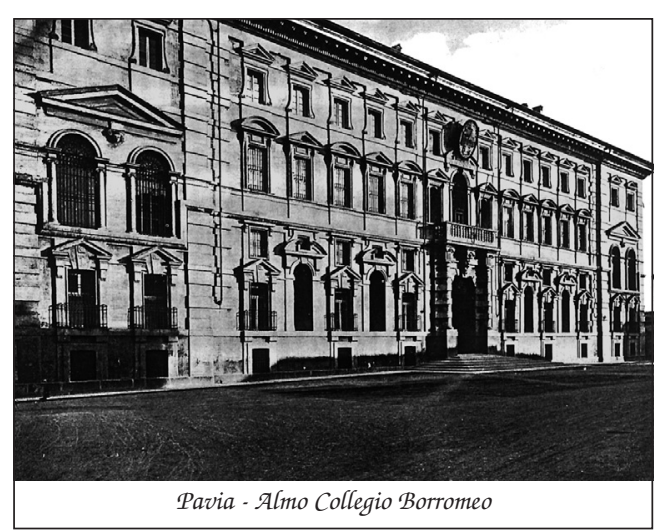

